\title{
Potential Utility of Multidetector Computed Tomography to Identify both Cardiac Embolic Sources and Coronary Artery Disease in Patients with Embolic Stroke
}

\author{
Kohichiro Iwasaki $^{a}$ Takeshi Matsumoto $^{a}$ Sanami Kawada $^{b}$ \\ Departments of a Cardiology and ${ }^{b}$ Neurology, Okayama Kyokuto Hospital, Okayama, Japan
}

\section{Key Words}

Cardiac embolic source $\cdot$ Coronary artery disease $\cdot$ Coronary computed tomographic angiography . Embolic stroke .

Multidetector computed tomography

\begin{abstract}
Objectives: Our objective was to study the potential utility of multidetector computed tomography (MDCT) to identify both cardiac embolic sources and coronary artery disease (CAD) in embolic-stroke patients. Methods: We performed MDCT for 184 patients with embolic stroke but without known CAD. Twenty-six patients had atrial fibrillation. We investigated the prevalence of the potential source of the embolism and the coronary characteristics. Results: Overall, 64 potential embolic sources were detected in 59 patients (32.1\%). Left atrial appendage thrombus, left ventricular thrombus and aortic atheroma were detected in 3.3, 0.5 and $15.8 \%$ of patients, respectively. Circulatory stasis and patent foramen ovale were detected in 8.7 and $6.5 \%$, respectively. As for coronary calcium score, only 47 patients $(25.5 \%)$ had a score of zero and $51(27.7 \%)$ had a score of $\geq 400$. Significant CAD was detected in 18 patients (9.8\%). One hundred and thirty-seven (74.5\%) had coronary plaques. The prevalence of positive remodeling, low-attenuation plaque, spot-
\end{abstract}

\section{KARGER}

E-Mail karger@karger.com

www.karger.com/crd ty calcification and a napkin-ring sign was 7.1, 1.6, 5.4 and $2.7 \%$, respectively. Importantly, only 34 patients $(13.0 \%)$ had no abnormalities detected by MDCT. Conclusions: Our results suggest that MDCT has potential to identify both cardiac embolic sources and CAD in patients with embolic stroke but without known CAD.

(c) 2015 S. Karger AG, Basel

Recently, ischemic stroke without carotid artery disease has emerged as a new coronary artery disease (CAD) risk equivalent [1]. An American Heart Association/ American Stroke Association scientific statement concludes that patients with atherosclerotic stroke should be included among those deemed to be at a high risk ( $\geq 20 \%$ over 10 years) of further atherosclerotic coronary events [2]. Coronary computed tomographic angiography (CCTA) is a noninvasive modality to evaluate CAD. Its ability to assess obstructive CAD has been demonstrated to be excellent compared with conventional coronary angiography $[3,4]$. Its ability to detect coronary plaques and to characterize plaque composition are also well-appreciated [5-7]. Several studies have investigated coronary characteristics of ischemic-stroke patients without known CAD [8-10]. However, these studies excluded embolic- 
stroke patients. Cardioembolic stroke can be expected to be associated with a higher likelihood of CAD, probably due to the underlying presence of cardiac disease $[11,12]$.

Transesophageal echocardiography (TEE) has been a gold standard for the detection of potential embolic sources in embolic-stroke patients $[13,14]$. However, TEE is a semi-invasive procedure with rare but potentially life-threatening complications. In addition, routine application of TEE is often limited in acute-stroke patients because of acute illness, mental changes, bleeding tendencies and the lack of cooperation by the patient. Moreover, echocardiography is dependent on the properties of the equipment and on the expertise of the technician. Recent studies show multidetector computed tomography (MDCT) to have as much diagnostic accuracy as TEE for the identification of potential embolic sources $[15,16]$. We studied the potential utility of MDCT to detect both cardiac embolic sources and CAD in embolicstroke patients without known CAD.

\section{Methods}

\section{Patients}

From January 2012 to May 2015, we performed MDCT for 184 patients with embolic stroke but without known CAD. A diagnosis of embolic stroke was performed according to the TOAST (Trial of Org 10172 in Acute Stroke Treatment) subtype classification system [17]. Exclusion criteria were: (1) death, (2) disabling stroke (i.e. a modified Rankin scale score $\geq 3$ ), (3) being older than 80 years, (4) having known CAD and (5) refusing to undergo MDCT. We investigated the source of embolism, and the coronary characteristics consisted of coronary calcium score (CCS) and the prevalence of significant stenosis, coronary plaque and high-risk plaque.

\section{$M D C T$}

All patients were scanned with a 64-MDCT scanner (SOMATOM Sensation 64 Cardiac, Siemens Medical Solutions, Erlangen, Germany). Patients with a heart rate $>70$ beats $/$ min received oral metoprolol $20 \mathrm{mg}$ before the 64-MDCT scan. To achieve coronary vasodilation, we administered sublingual nitroglycerin 0.8 $\mathrm{mg}$ before the scan.

A native scan without contrast dye was performed to determine the total calcium burden of the coronary tree (sequential scan with $32 \times 0.6 \mathrm{~mm}$ collimation, tube current $60 \mathrm{mAs}$ at $120 \mathrm{kV}$ ). Contrast-enhanced CT angiography data were acquired with the use of a spiral scan with $32 \times 0.6 \mathrm{~mm}$ collimation, $330-\mathrm{ms}$ gantry rotation, a pitch of 0.2 and tube voltage of $120 \mathrm{kV}$. Contrast agent (50-60 $\mathrm{ml} ; 370 \mathrm{mg}$ iodine $/ \mathrm{ml}$ ) was injected intravenously $(4.0 \mathrm{ml} / \mathrm{s})$ followed by a $30-\mathrm{ml}$ saline chaser. Transaxial images were reconstructed using an ECG-gated half-scan reconstruction algorithm (temporal resolution $164 \mathrm{~ms}$ ) and kernel B30f. Late-phase imaging, using prospective electrocardiographic gating for added images of the left atrial appendage (LAA), was obtained 1 min after starting the injection of contrast media.

\section{MDCT Image Interpretation}

We studied the potential cardiac sources of embolism according to the TOAST classification [17]. A thrombus was defined as a filling defect that appeared on both early- and late-phase images with an oval or round shape and a late-phase ratio in Hounsfield units (HU) of the LAA to the ascending aorta (AA) of $<0.5[18,19]$. Circulatory stasis was defined as a filling defect that appeared as a triangular shape in the LAA only on the early-phase images but was absent on the late-phase images, and an LAA/AA ratio of $>0.5$. Patent foramen ovale (PFO) was defined as the presence of a contrast jet from the left to the right atrium toward the inferior vena cava with a channel-like appearance of the interatrial septum [20]. Aortic atheroma was defined as thrombus, ulceration and a discrete, nonmobile lesion $>4 \mathrm{~mm}$ in the AA or the aortic arch.

\section{CCTA Image Interpretation}

CT data sets were transferred to an offline workstation (Aquarius NetStation, TeraRecon Inc., San Mateo, Calif., USA) for image analysis. The CCS for all patients was calculated with dedicated software, which calculates the total amount of calcium on the basis of the number, areas and peak HU of the detected calcified lesions [21].

The contrast-enhanced CT angiography data were evaluated by 2 reviewers blinded to the clinical characteristics of the patients, using maximum intensity and curved multiplanar reconstruction techniques along multiple longitudinal axes and also transversely. Standard display settings were used for the evaluation of the contrast-enhanced 64-MDCT scans (window width $800 \mathrm{HU}$; window center $250 \mathrm{HU}$ ). We defined calcified plaque as any structure with a density $>130 \mathrm{HU}$ which could be visualized separately from the coronary lumen, assigned to the coronary artery wall and was identified in at least 2 independent planes [22]. We defined noncalcified plaque as any structure which could be assigned to the coronary artery wall, had a CT density less than the contrast-enhanced coronary lumen but greater than the surrounding connective tissue and was identified in at least 2 independent planes [22]. We defined positive remodeling as a diameter at the plaque site $>10 \%$ larger than that of the reference segment [23]. We defined spotty calcification as $<3 \mathrm{~mm}$ in size on curved multiplanar reformation images and occupying only 1 side on cross-sectional images [24]. In noncalcified plaque or plaque with spotty calcification, we measured the lowest CT number of 5 areas of minimum region of interest using the axial image. We defined low-attenuation plaque as $<30 \mathrm{HU}$ on CT [23]. We defined a napkin-ring sign as the presence of a ring of high attenuation around certain coronary artery plaques and the CT attenuation of a ring presenting higher than those of the adjacent plaques and $<130 \mathrm{HU}$ [25]. We defined highrisk plaque as a plaque with positive remodeling, low-attenuation plaque, spotty calcification or a napkin-ring sign. Two reviewers identified coronary segments and classified the segments as normal (smooth, parallel or tapering borders), as having nonsignificant stenosis (luminal irregularities or $<50 \%$ stenosis) or with significant stenosis ( $>50 \%$ stenosis).

Informed consent for clinical procedures and research protocol was received from all patients studied. The study was approved by an institutional review board.

\section{Statistical Analysis}

Continuous variables are expressed as mean \pm SD. Discrete variables are expressed as counts or percentage. 
Table 1. Characteristics of 184 patients

\begin{tabular}{lc}
\hline Characteristics & $69.1 \pm 13.3$ \\
\hline Age, years & $122: 62$ \\
Sex, M:F & \\
Risk factors & $140(76.1)$ \\
$\quad$ Hypertension & $108(58.7)$ \\
Hyperlipidemia & $38(20.7)$ \\
Diabetes & $36(19.6)$ \\
Obesity & $30(16.3)$ \\
Smoking & $6(3.3)$ \\
Atrial fibrillation & $20(10.9)$ \\
Paroxysmal & \\
Chronic & $130.0 \pm 44.3$ \\
Laboratory data & $5.9 \pm 1.1$ \\
BS, mg/dl & $203.7 \pm 43.8$ \\
HbAlc, \% & $137.2 \pm 86.2$ \\
TC, mg/dl & $53.6 \pm 16.3$ \\
TG, mg/dl & $123.5 \pm 38.3$ \\
HDL-C, mg/dl & \\
LDL-C, mg/dl &
\end{tabular}

Values are expressed as $\mathrm{n}(\%)$ or means $\pm \mathrm{SD}$. BS = Blood sugar; $\mathrm{HbA} 1 \mathrm{c}=$ hemoglobin A1c; HDL-C = high-density lipoprotein cholesterol; LDL-C = low-density lipoprotein cholesterol; $\mathrm{TC}=$ total cholesterol; $\mathrm{TG}=$ triglyceride.

\section{Results}

The clinical characteristics of the studied patients are shown in table 1 . Twelve patients had chronic atrial fibrillation and the other 6 had paroxysmal atrial fibrillation. Table 2 shows the prevalence of potential cardiac embolic sources. Overall, 64 potential embolic sources were detected in 59 patients $(32.1 \%)$. LAA thrombus was detected in 6 patients: 3 with sinus rhythm, 2 with paroxysmal atrial fibrillation and 1 with chronic atrial fibrillation. Circulatory stasis was detected in 16 patients: 3 with paroxysmal atrial fibrillation and 13 with chronic atrial fibrillation.

Table 3 shows the CCS and coronary artery characteristics of the studied patients. Only $47(25.5 \%)$ had a zero CCS and 51 (27.7\%) had a high-risk CCS defined as CCS $\geq 400$. Significant CAD was detected in 18 patients $(9.8 \%)$. Surprisingly, 8 patients had chronic total occlusion in at least 1 coronary artery, and 7 had multivessel CAD. The prevalence of high-risk plaque was $8.7 \%$. Importantly, only $34(13.0 \%)$ had no abnormalities detected by MDCT. Figures 1-4 show a representative case of LAA thrombus, $\mathrm{PFO}$, aortic atheroma and CAD, respectively.
Table 2. Potential cardiac sources of embolism in 184 patients

\begin{tabular}{lc}
\hline & $\mathrm{n}(\%)$ \\
\hline High-risk sources & \\
LAA thrombus & $6(3.3)$ \\
Aortic atheroma & $29(15.8)$ \\
LV thrombus & $1(0.5)$ \\
Medium-risk sources & $16(8.7)$ \\
Circulatory stasis & $12(6.5)$ \\
PFO & $64(34.8)$ \\
\hline Total & \\
\hline LV = Left ventricle.
\end{tabular}

Table 3. CCS and coronary artery characteristics of 184 patients

\begin{tabular}{lc}
\hline CCS, $\mathrm{n}(\%)$ & \\
0 & $47(25.5)$ \\
$1-99$ & $42(22.8)$ \\
$100-399$ & $44(24.0)$ \\
$400-999$ & $27(14.7)$ \\
$\geq 1,000$ & $24(13.0)$ \\
Characteristics, $\mathrm{n}(\%)$ & \\
Significant stenosis & $18(9.8)$ \\
Any plaque & $137(74.5)$ \\
Any high-risk plaque & $16(8.7)$ \\
PR & $13(7.1)$ \\
LAP & $3(1.6)$ \\
Spotty calcification & $10(5.4)$ \\
Napkin-ring sign & $5(2.7)$ \\
Segments, means \pm SD & \\
Including any plaque & $3.0 \pm 2.4$ \\
Including Ca plaques & $2.7 \pm 2.4$ \\
Including non-Ca plaque & $0.2 \pm 0.6$ \\
\hline
\end{tabular}

$\mathrm{Ca}=$ Calcified; LAP = low-attenuation plaque; $\mathrm{PR}=$ positive remodeling.

\section{Discussion}

Our results showed that in embolic-stroke patients, MDCT detected potential embolic sources in 32\%, highrisk CCS in $28 \%$, significant coronary stenosis in $10 \%$ and high-risk plaque in $9 \%$. Importantly, only $13 \%$ of the patients had no abnormalities. Only 1 other study investigated the prevalence of both cardiac embolic sources and CAD in patients with embolic stroke [12].

There are several studies which compared MDCT and TEE for the detection of cardiac sources in embolic-stroke 

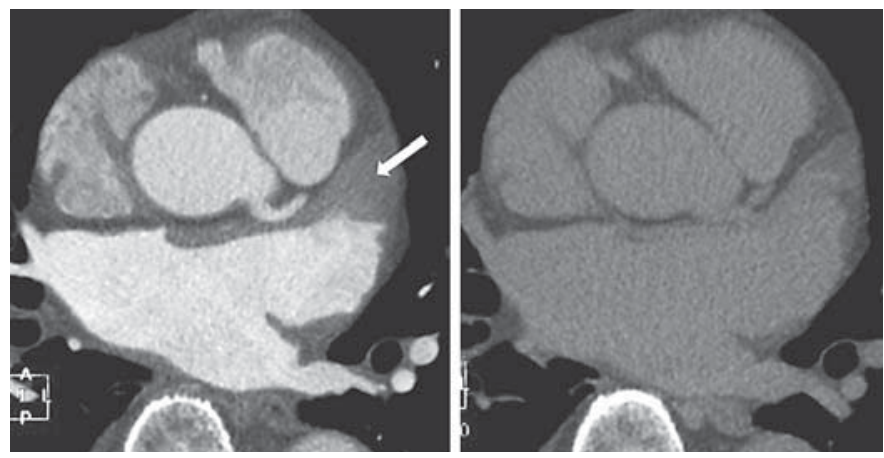

Fig. 1. LAA thrombus. Axial MDCT images from a 63-year-old man demonstrate a filling defect (arrow) on an early-phase image. The late-phase ratio LAA/AA (in HU) was 0.18 .
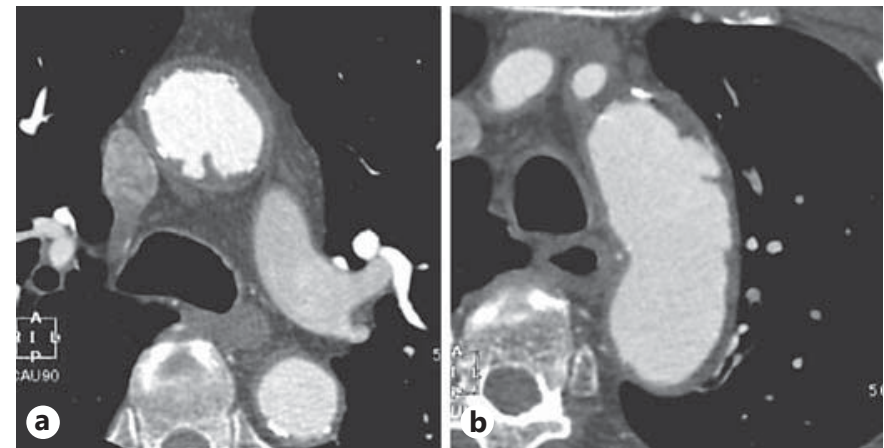

Fig. 3. Aortic atheroma. Axial MDCT images from a 74-year-old man demonstrate ulceration and a discrete nonmobile lesion in the AA (a) and the aortic $\operatorname{arch}(\mathbf{b})$.

patients. Ko et al. [15] performed MDCT and TEE in 75 patients. MDCT identified a high-risk, intracardiac embolic source in 8 and an extracardiac source in 20, while TEE found an intracardiac source in 1 and an extracardiac source in 7. Kim et al. [12] found that cardioaortic sources of cerebral embolism were more frequently detected in the MDCT period than in the TEE period (18.1 vs. $6.6 \%, \mathrm{p}<0.001)$. Hur et al. [16] performed MDCT and TEE in 137 patients with a recent episode of stroke. They found potential cardiac sources in 80 patients (58.4\%) including LAA/left atrium thrombus (8.8\%), aortic atheroma (22.6\%), circulatory stasis (10.9\%) and PFO (10.2\%). Their results are similar to ours.
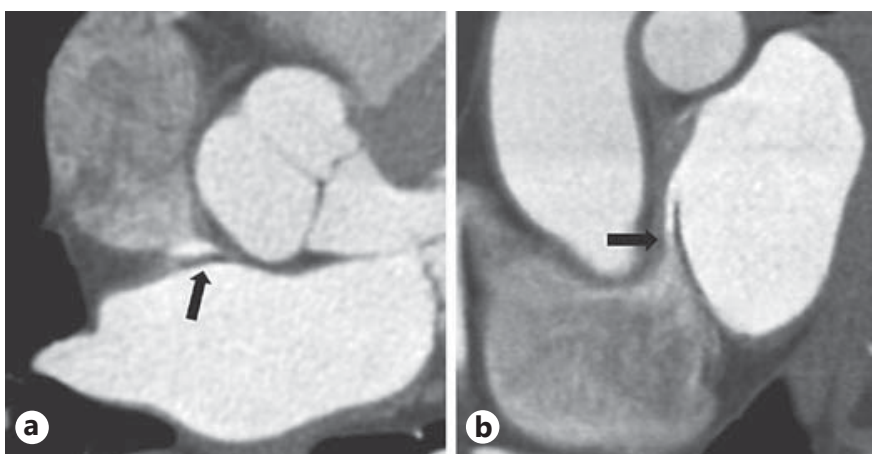

Fig. 2. PFO. Axial (a) and oblique sagittal (b) MDCT images from a 61-year-old woman demonstrate the channel-like appearance of the interatrial septum (arrow in b) and the contrast jet from the left to the right atrium towards the inferior vena cava (arrows).
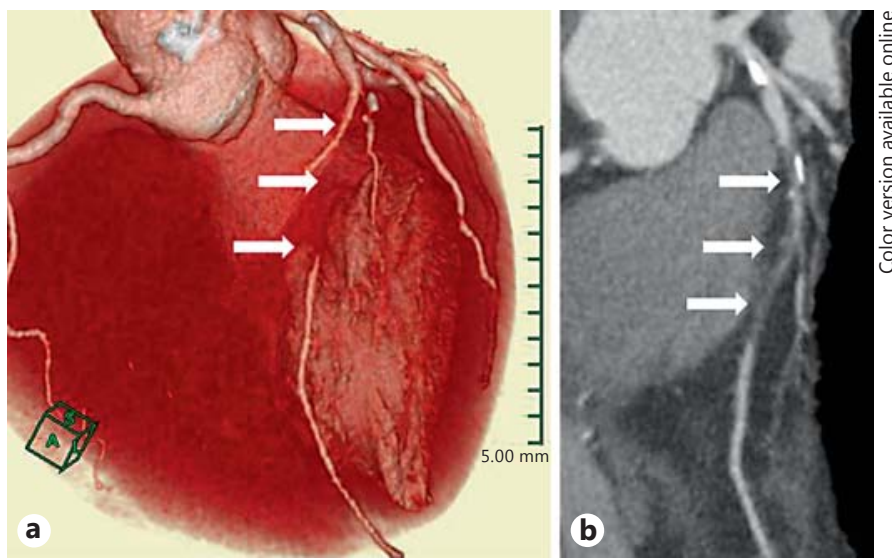

Fig. 4. CAD. CCTA: a volume-rendering image (a) and a curved multiplanar reconstruction image (b) show chronic total occlusion of the mid-portion of the left anterior descending coronary artery (arrows).

The distinction between LAA thrombus and circulatory stasis is important and was established by the study of Kim et al. [18]. Furthermore, a meta-analysis showed that in a subanalysis of studies in which delayed imaging was performed, the diagnostic accuracy significantly improved to a mean weighted sensitivity and specificity of 100 and $99 \%$, respectively, but the positive predictive value and negative predictive value increased to 92 and $100 \%$, respectively. The accuracy for this technique was $99 \%$ [19]. Thus we used the definition of Kim et al. [18].

Previous studies investigated the prevalence of CAD in patients with ischemic stroke or transient ischemic attack but without known CAD [8-10]. They showed that 20- 
$40 \%$ of stroke patients had asymptomatic significant CAD, but they excluded patients with embolic stroke. Only a few reports include patients with embolic stroke. Yoon et al. [11] performed CCTA in 175 patients suspected to have had embolic stroke/transient ischemic attack; atherosclerotic plaques were identified in 60 and $21 \%$ had occult CAD with stenosis $\geq 50 \%$ of the diameter. Kim et al. [12] found significant CAD in $36 \%$ of 200 patients with ischemic stroke but without known atrial fibrillation or CAD. Our results showed that $75 \%$ of patients had coronary plaques and $10 \%$ had significant stenosis. Surprisingly, $8 / 18$ of our patients had chronic total occlusion.

We also measured CCS. Coronary artery calcification signifies the presence of coronary atherosclerosis, and a strong linear correlation exists between total coronary artery atherosclerotic plaque burden and the extent of coronary artery calcification $[26,27]$. CCS has been found to be the most powerful predictor of cardiac events, providing independent and incremental information over risk factor-based assessments of asymptomatic patients [28, 29]. Thus, the measurement of CCS would have an additional value for the risk stratification of asymptomatic CAD in stroke patients. Our results showed that only one quarter of the embolic-stroke patients had a CCS of zero and another quarter had high-risk CAD.

Recent studies demonstrate that CCTA characteristics of plaques associated with vulnerability are positive re- modeling, low-attenuation plaque and spotty calcification $[23,24]$. In addition, a napkin-ring sign appears to be an indication of a high-risk coronary plaque [25]. Thus, we defined high-risk plaque as positive remodeling, low-attenuation plaque, spotty calcification or napkinring sign. The prevalence of high-risk plaque was about $10 \%$; such patients should receive high-intensity statin therapy to prevent coronary events.

There are some limitations in our study. First, the number of patients was relatively small. Second, we excluded patients who had died, who had disabling stroke and who declined MDCT. It is likely that, because of these exclusions, the coronary characteristics of embolic-stroke patients were underestimated.

In conclusion, our results suggest that MDCT has potential utility to identify both cardiac embolic sources and CAD in patients with embolic stroke but without known CAD. Importantly, our results showed that only $13 \%$ of embolic-stroke patients had no abnormalities detected by MDCT. Compared with semi-invasive TEE, MDCT has the advantage of being noninvasive and it has the ability to detect and characterize CAD.

\section{Conflict of Interest}

There were no conflicts of interest.

\section{References}

1 Dhamoon MS, Elkind MSV: Inclusion of stroke as an outcome and risk equivalent in risk scores for primary and secondary prevention of vascular disease. Circulation 2011;121: 2071-2078

2 Lackland DT, Elkind MS, D’Agostino R Sr, Dhamoon MS, Goff DC Jr, Higashida RT, McClure LA, Mitchell PH, Sacco RL, Sila CA, Smith SC Jr, Tanne D, Tirschwell DL, Touzé E, Wechsler LR; on behalf of the American Heart Association Stroke Council, Council on Epidemiology and Prevention, Council on Cardiovascular Radiology and Intervention, Council on Cardiovascular Nursing, Council on Peripheral Vascular Disease, and Council on Quality of Care and Outcomes Research: Inclusion of Stroke in Cardiovascular Risk Prediction Instruments: A Statement for Healthcare Professionals From the American Heart Association/American Stroke Association. Stroke 2012;43:1998-2027.
3 Hamon M, Biondi-Zoccai GG, Malagutti P, Agostoni P, Morello R, Valgimigli M, Hamon M: Diagnostic performance of multislice spiral computed tomography of coronary arteries as compared with conventional invasive coronary angiography: a meta-analysis. J Am Coll Cardiol 2006;48:1896-1910.

4 Abdulla J, Abildstrom SZ, Gotzsche O, Christensen E, Kober L, Torp-Pedersen C: 64-Multislice detector computed tomography coronary angiography as potential alternative to conventional coronary angiography: a systematic review and meta-analysis. Eur Heart J 2007;28:3042-3050.

5 Achenbach S, Moselewski F, Ropers D, Ferencik M, Hoffmann U, MacNeill B, Pohle K, Baum U, Anders K, Jang IK, Daniel WG, Brady TJ: Detection of calcified and noncalcified coronary atherosclerotic plaque by contrast-enhanced, submillimeter multidetector spiral computed tomography. A segmentbased comparison with intravascular ultrasound. Circulation 2004;109:14-17.
6 Leber AW, Knez A, von Zieger F, Becker A, Nikolaou K, Paul S, Wintersperger B, Reiser M, Becker CR, Steinbeck G, Boekstegers P: Quantitation of obstructive and nonobstructive coronary lesions by 64-slice computed tomography. A comparative study with quantitative coronary angiography and intravascular ultrasound. J Am Coll Cardiol 2005;46: $147-154$.

7 Carrascosa PM, Capuñay CM, Garcia-Merletti P, Carrascosa J, Garcia MJ: Characterization of coronary atherosclerotic plaques by multidetector computed tomography. Am J Cardiol 2006;97:598-602.

8 Yoo J, Yang JH, Choi BW, Kim YD, Nam HS, Choi HY, Lee HS, Cha MJ, Choi D, Nam CM, Jang Y, Lee DH, Kim J, Heo JH: The frequen$\mathrm{cy}$ and risk of preclinical coronary artery disease detected using multichannel cardiac computed tomography in patients with ischemic stroke. Cerebrovasc Dis 2012;33:286294. 
9 Hoshino A, Nakamura T, Enomoto S, Kawahito H, Kurata H, Nakahara Y, Ijichi T: Prevalence of coronary artery disease in Japanese patients with cerebral infarction. Circ J 2008; 72:404-408.

10 Calvet D, Touze E, Varenne O, Sablayrolles JL, Weber S, Mas JL: Prevalence of asymptomatic coronary artery disease in ischemic stroke patients. The PRECORIS study. Circulation 2010;121:1623-1629.

11 Yoon YE, Chang HJ, Cho I, Jeon KH, Chun EJ, Choi SI, Bae HJ, Rivera JJ, Nasir K, Blumenthal RS, Lim TH: Incidence of subclinical coronary atherosclerosis in patients with suspected embolic stroke using cardiac computed tomography. Int J Cardiovasc Imaging 2011:27:1035-1044.

12 Kim SJ, Choe YH, Park SJ, Kim GM, Chung CS, Lee KH, Bang OY: Routine cardiac evaluation in patients with ischaemic stroke and absence of known atrial fibrillation or coronary heart disease: transthoracic echocardiography vs. multidetector cardiac computed tomography. Eur J Neurol 2012;19:317323.

13 Pearson AC, Labovitz AJ, Tatineni S, Gomez CR: Superiority of transesophageal echocardiography in detecting cardiac source of embolism in patients with cerebral ischemia of uncertain etiology. J Am Coll Cardiol 1991; 17:66-72.

14 Cujec B, Polasek P, Voll C, Shuaib A: Transesophageal echocardiography in the detection of potential cardiac source of embolism in stroke patients. Stroke 1991;22:727-733.

15 Ko SB, Choi SI, Chun EJ, Ko Y, Park JH, Lee SJ, Lee J, Han MK, Bae HJ: Role of cardiac multidetector computed tomography in acute ischemic stroke: a preliminary report. Cerebrovasc Dis 2010;29:313-320.
16 Hur J, Kim YJ, Lee HJ, Ha JW, Heo JH, Choi EY, Shim CY, Kim TH, Nam JE, Choe KO, Choi BW: Cardiac computed tomographic angiography for detection of cardiac sources of embolism in stroke patients. Stroke 2009; 40:2073-2078.

17 Adams HP Jr, Bendixen BH, Kappelle LJ, Biller J, Love BB, Gordon DL, Marsh EE 3rd: Classification of subtype of acute ischemic stroke. Definitions for use in a multicenter clinical trial. TOAST. Trial of Org 10172 in Acute Stroke Treatment. Stroke 1993;24:3541.

18 Kim SC, Chun EJ, Choi SI, Lee SJ, Chang HJ, Han MK, Bae HJ, Park JH: Differentiation between spontaneous echocardiographic contrast and left atrial appendage thrombus in patients with suspected embolic stroke using two-phase multidetector computed tomography. Am J Cardiol 2010;106:1174-1181.

19 Romero J, Husain SA, Kelesidis I, Sanz J, Medina HM, Garcia MJ: Detection of left atrial appendage thrombus by cardiac computed tomography in patients with atrial fibrillation: a meta-analysis. Circ Cardiovasc Imaging 2013;6:185-194.

20 Kim YJ, Hur J, Shim CY, Lee HJ, Ha JW, Choe KO, Heo JH, Choi EY, Choi BW: Patent foramen ovale: diagnosis with multidetector CT - comparison with transesophageal echocardiography. Radiology 2009;250:61-67.

21 Agatston AS, Janowitz WR, Hildner FJ, Zusmer NR, Viamonte M Jr, Detrano R: Quantification of coronary artery calcium using ultrafast computed tomography. J Am Coll Cardiol 1990;15:827-832.

22 Ferencik M, Nieman K, Achenbach S: Noncalcified and calcified coronary plaque detection by contrast-enhanced multi-detector computed tomography: a study of interobserver agreement. J Am Coll Cardiol 2006;47: 207-209.

23 Motoyama S, Kondo T, Sarai M, Sugiura A, Harigaya $\mathrm{H}$, Sato $\mathrm{T}$, Inoue $\mathrm{K}$, Okumura $\mathrm{M}$, Ishii J, Anno H, Virmani R, Ozaki Y, Hishida H, Narula J: Multislice computed tomographic characteristics of coronary lesions in acute coronary syndromes. J Am Coll Cardiol 2007; 50:319-326.
24 Ehara S, Kobayashi Y, Yoshiyama M, Shimada K, Shimada Y, Fukuda D, Nakamura Y, Yamashita H, Yamagishi H, Takeuchi K, Naruko T, Haze K, Becker AE, Yoshikawa J, Ueda M: Spotty calcification typifies the culprit plaque in patients with acute myocardial infarction: an intravascular ultrasound study. Circulation 2004;110:3424-3429.

25 Otsuka K, Fukuda S, Tanaka A, Nakanishi K, Taguchi H, Yoshikawa J, Shimada K, Yoshiyama M: Napkin-ring sign on coronary CT angiography for the prediction of acute coronary syndrome. JACC Cardiovasc Imaging 2013;6:448-457.

26 Rumberger JA, Simons DB, Fitzpatrick LA, Sheedy PF, Schwartz RS: Coronary artery calcium area by electron-beam computed tomography and coronary atherosclerotic plaque area. A histopathologic correlative study. Circulation 1995;92:2157-2162.

27 Sangiorgi G, Rumberger JA, Severson A, Edwards WD, Gregorie J, Fitzpatrick LA, Schwartz RS: Arterial calcification and not lumen stenosis is highly correlated with atherosclerotic plaque burden in humans: a histologic study of 723 coronary artery segments using nondecdalcifying methodology. J Am Coll Cardiol 1998;31:126-133.

28 Erbel R, Möhlenkamp S, Moebus S, Schmermund A, Lehmann N, Stang A, Dragano N, Grönemeyer D, Seibel R, Kälsch H, BröckerPreuss M, Mann K, Siegrist J, Jöckel KH: Coronary risk stratification, discrimination, and reclassification improvement based on quantification of subclinical coronary atherosclerosis: the Heinz Nixdorf Recall Study. J Am Coll Cardiol 2010;56:1397-1406.

29 Yeboah J, McClelland RL, Polonsky TS, Burke GL, Sibley CT, O'Leary D, Carr JJ, Goff DC, Greenland P, Herrington DM: Comparison of novel risk markers for improvement in cardiovascular risk assessment in intermediaterisk individuals. JAMA 2012;308:788-795. 